\title{
Dynamics of Active Regions Revealed by Tracking of Doppler Features
}

\author{
M. Švanda, M. Sobotka, M. Klvaňa, and V. Bumba
}

\begin{abstract}
We investigate the large-scale horizontal dynamics of active regions in the 23rd solar cycle. The large-scale horizontal velocity fields were measured applying the local correlation tracking (LCT) algorithm to the processed high-cadence full-resolution full-disc MDI Dopplergrams. We performed the selection of NOAA active regions in the available dataset and followed their individual evolution in time. The statistical study of this sample gives us a unique opportunity to study the dynamics of active regions at various stages of their evolution. In few cases, we found behavior that is consistent with the dynamical disconnection of sunspots from the magnetic roots.
\end{abstract}

\section{Introduction}

The dynamics of active regions in the solar photosphere and in the close subphotospheric layers is closely related to the solar dynamo process. The magnetic flux emerges from beneath the surface and forms the sunspots and other active phenomena. At some point, the magnetic field starts to disperse, the sunspots disappear, and the active region dies, forming surge-like structures of the trailing polarity expanding to the solar poles, where they contribute to the solar field reversals.

The measurements of the dynamical behavior of active regions can bring new insights in what is going on with the magnetic field under the surface. In particular, some ideas supported by the theory (Fan et al. 1994; Schüssler and Rempel 2005) suggested that at some point, bipolar magnetic regions may disconnect from their magnetic roots and form an isolated island-like feature. The mechanism is based on the buoyant upflow of plasma along the field lines. Such flows arise in the upper

\author{
M. Švanda (凶) \\ Ondřejov Observatory, Academy of Sciences, Ondřejov, Czech Republic \\ and \\ Astronomical Institute, Charles University, Prague, Czech Republic \\ M. Sobotka, M. Klvaňa, and V. Bumba \\ Ondřejov Observatory, Academy of Sciences, Ondřejov, Czech Republic
}


part of a rising flux loop during the final phases of its buoyant ascent towards the surface. The combination of the pressure build-up by the upflow and the cooling of the upper layers of an emerged flux tube by radiative losses at the surface leads to a progressive weakening of the magnetic field in several mega-meter depth. When the field strength has become sufficiently low, convective motions ablate the flux tube into thin, passively advected flux fragments, thus providing a dynamical disconnection of the emerged part from its parent magnetic structure. This instant should be observed as the change in the dynamical regime, because the floating island does not reflect the deep dynamics anymore. In this contribution, we investigate this issue in a more detail.

\section{The Data and Method}

In recent papers (e.g., Švanda et al. 2006), we introduced a method to measure the large-scale dynamics in the solar photosphere. It is based on tracking supergranular structures in full-disk Dopplergrams. Its application allows us to compute 24-h averaged horizontal flow fields with a resolution of $60^{\prime \prime}$ at a noise level of $15 \mathrm{~m} \mathrm{~s}^{-1}$. In the magnetized regions, this method makes it possible to measure the apparent motion of supergranular-scale magnetic features.

In our set of 1,004 flow maps, we identified 564 active regions, of which 522 were observed in more than one flow map and 194 emerged less than $60^{\circ}$ from the central meridian. From this sub-set we selected 69 bipolar regions that survived at least 4 days within central meridian distance less than $60^{\circ}$. This drastic reduction of the sample was necessary to avoid any possible bias.

For each active region in the sample, we computed the large-scale flow field in the area, the average rotation speed, the magnetic field area (with threshold of $100 \mathrm{G}$ ), and the mean magnetic field intensities in both leading and trailing polarities, sampled per $12 \mathrm{~h}$. We investigated the change in the dynamics that may be connected to the effect of disconnection from the magnetic roots. The expected change should display sudden deceleration because the radial gradient of the mean rotation is negative in nearby subphotospheric layers.

\section{Results and Conclusions}

We found that $75 \%$ of the active regions in the selected sample show the characteristic change in dynamics. These active regions display a sudden decrease in their proper rotation rate, typically within 3 days after their emergence. Dynamical disconnection from their magnetic roots is a natural explanation for this behavior. Our study suggests that this phenomenon is quite common among bipolar active regions.

We observe a systematic phase shift between the time of the dynamical regime change and the time of the maximum in the area. We interpret this as the time during 

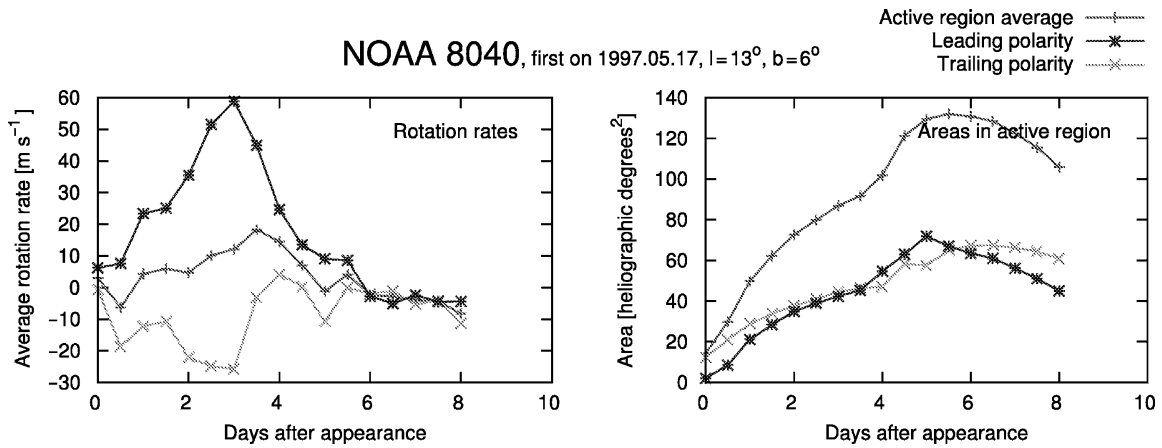

Fig. 1 The evolution of the mean rotation speed with respect to the long-term background, active region area, and the mean field in active region NOAA 8040. This is an example of the active region displaying the signature of dynamical disconnection

which the magnetic island rises from its parent magnetic structure to the surface after the disconnection. After this takes place, no more magnetic field is fed into the region and the region starts to diminish.

The systematic shift is $1.67 \pm 0.72$ days. Assuming a $1-\mathrm{kG}$ fluxtube emerging at the Alfvén speed, the theoretical shift is 180 days if the fluxtube emerges from the bottom of the convection zone and 7 days when emerging from a subsurface shear layer at $0.95 \mathrm{R}_{\odot}$. The measured lag corresponds to the depth $17 \pm 4 \mathrm{Mm}$. The Alfvén speed computation is based on model S of Christensen-Dalsgaard et al. (1996). The actual rise speed of an expanding fluxtube should not differ from the Alfvén speed in order of magnitude. The computation shows that the disconnection cannot occur deeper than some $40 \mathrm{Mm}$ for a $4-\mathrm{kG}$ fluxtube to reproduce the observed behavior. Although these numbers represent only a rough estimate, our results show that the disconnection takes place just a few Mega-meter below the photosphere.

We conclude that dynamical disconnection is an important part of the life of bipolar active regions. Our results favor a sub-surface shear layer as the location of origin for surface magnetic activity.

Acknowledgment This work was supported by the Grant Agency of Academy of Sciences of the Czech Republic under grant No. IAA30030808 and by research project No. MSM0021620860 of the Ministry of Education of the Czech Republic.

\section{References}

Christensen-Dalsgaard, J., Dappen, W., Ajukov, S. V., et al. 1996, Science, 272, 1286

Fan, Y., Fisher, G. H., McClymont, A. N. 1994, ApJ, 436, 907

Schüssler, M., Rempel, M. 2005, A\&A, 441, 337

Švanda, M., Klvaňa, M., Sobotka, M. 2006, A\&A, 458, 301 